\title{
Classification of Satellite Images through Gabor Filter using SVM
}

\author{
Manali Jain \\ M.Tech (CSE) Scholar \\ ABES Engineering College, Ghaziabad
}

\author{
Amit Sinha \\ Associate Professor \\ ABES Engineering College, Ghaziabad
}

\begin{abstract}
The classification of satellite image has various issues including image quality. The aim of classifying satellite image is to provide better understanding of image. The classification of image depends on various features. the present paper focuses on five categories of satellite image such as residential area, agriculture, desert, mountain and forest. The objective of this paper is to study the use of texture, color, shape as an image feature for pattern retrieval. We propose the novel approach to extract features of image through Gabor filter feature vector i.e color, texture, shape and classification of image through support vector machine (SVM) with the use of Gaussian radial basis kernel function. The proposed methodology gives the better accuracy up to $98.5 \%$ over DCT Gabor based classification. The work is useful and may be embedded with different applications like residential purposes and government planning commission and national environment mission etc.
\end{abstract}

\section{Keywords}

Satellite Images, Gabor filter feature vector, DCT, Support Vector Machine.

\section{INTRODUCTION AND LITERATURE SURVEY}

In image processing applications, Gabor filter is the successful technique for construction of image features and Gabor features selection. For example, in iris recognition Daugman's iris code [1] is the best and gives ideal standards for the iris recognition. In face recognition [2] and fingerprint matching [3] the performance of Gabor features are appreciable. Gabor features extract local pieces of information which are then combined to recognize an object or region of interest. The dynamic link architecture (DLA) was introduces by the Lades et al. [4]. He introduced the concept of "Gabor jets". Further this concept is extended by Wiskott et al. and gave the method of elastic bunch graph matching (EBGM). This technique is used for detection and recognition of objects like human faces [5]. They gave the model of the simple cell response function in the mammalian visual cortex [6] and Gabor filters is the main motivation, and this old adage is continuously resurrected [7]. Features based on Gabor filter responses perform remarkably well in many modern problems and applications of computer vision and image processing.

One challenging problem is the extracting information of image data which are based on pictorial queries. Recently the multimedia database is come into existence and digital libraries make this problem more important. While in image searching the, the manual image comment can be used to a certain extent. Manual image search approach is feasible on the large database. This is a questionable issue. In some cases, such as satellite image patterns, simple textual descriptions can be arguable and continuously insufficient for database search.
The purpose of this paper is to use of texture, color and shape of the image as an image feature which is used for the pattern retrieval. An image can be considered as a pattern produced by arranging together small pieces of different texture, color and shape regions and the image features associated with these regions can be used for search and retrieval. A typical query could be a region of interest provided by the user such as type of image in a satellite image.

Texture analysis has a long history and texture analysis algorithms range from using random field models to multi resolution filtering techniques such as the wavelet transform. Texture features are used by many researchers for the retrieval of patterns [8]. In this paper we focus on the Gabor filter based feature extraction and after feature extraction, feature selection is applied for the training of these features with the help of well known classification technique called support vector machine (SVM). The use of Gabor filters in extracting textured image features is motivated by various factors. The Gabor representation has been shown to be optimal in the sense of minimizing the joint two-dimensional uncertainty in space and frequency [9]. . Gabor features have been used in several images. These filters can be considered as orientation and scale tunable edge and line (bar) detectors, and the statistics of these micro features in a given region are often used to characterize the underlying texture, color and shape information

\section{FEATURE EXTRACTION AND FEATURE SELECTION}

The core of Gabor filter based feature extraction is the $2 \mathrm{D}$ Gabor filter function [8]:

$$
\begin{gathered}
\varphi(x, y)=\frac{f^{2}}{\pi \gamma \mu} e^{-\left(\frac{f^{2}}{\gamma^{2}} x^{\prime 2}+\frac{f^{2}}{\gamma^{2}} y^{\prime 2}\right) e^{j 2 \pi f x^{\prime}}} \\
x^{\prime}=x \cos \theta+y \sin \theta \\
y^{\prime}=-x \sin \theta+y \cos \theta
\end{gathered}
$$

This function has the following analytical form in the frequency domain

$$
\begin{gathered}
\varphi(u, v)=e^{-\frac{\pi^{2}}{f^{2}\left(y^{2}\left(u^{\prime}-f\right)^{2}+n^{2} v^{\prime 2}\right)}} \\
u^{\prime}=u \cos \theta+v \sin \theta \\
v^{\prime}=-u \sin \theta+v \cos \theta(2)
\end{gathered}
$$

In the frequency domain (2) the function is a single realvalued Gaussian centered at $\mathrm{f}$. The Gabor filter in (1) and (2) is a simplified version of the general $2 \mathrm{D}$ form devised by Daugman [6] from the Gabor's original 1D "elementary function".

Gabor features are constructed from responses of Gabor filters in (1) or (2) by using multiple filters on several 
frequencies fm and orientations $\theta$ n. Frequency in this case corresponds to scale information and is thus drawn from [10]

$f_{m}=k^{-m} f_{\text {max }}, \mathrm{m}=\{0, \ldots, \mathrm{M}-1\}$

Where $\mathrm{fm}$ is the mth frequency, $\mathrm{fO}=\mathrm{fmax}$ is the highest frequency desired, and $\mathrm{k}>1$ is the frequency scaling factor. The filter orientations are drawn from [10]

$\theta_{n}=\frac{n 2 \pi}{N}, \mathrm{n}=\{0, \ldots, \mathrm{N}-1\}$

Where $\theta \mathrm{n}$ is the nth orientation and $\mathrm{N}$ is the total number of orientations.

Raw features are the complex-valued responses of a set of multi-resolution Gabor filters as illustrated in Fig. 2. Using a classifier, as simple as Gaussian mixture models in the facial feature detector in [14], the features can be effectively used to detect and recognize complex real world structures in images.

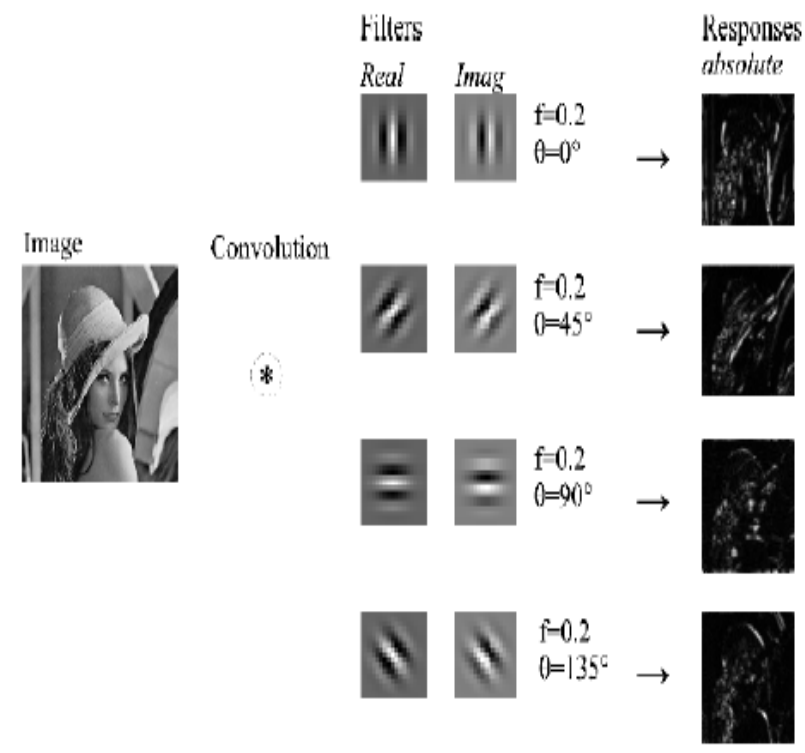

Figure 2. Feature extraction using a bank of Gabor filters.

\section{USE OF SVM IN CLASSIFICATION OF SATELLITE IMAGE}

After feature extraction, we employ Support Vector Machines (SVM) for multi-object classification. Support Vector Machines (SVM) [15] is a statistical learning based classification system where the basic idea is to find a hyper plane which separates the d-dimensional data perfectly into its two classes.

The input satellite image is classified into five categories using a SVM. The classifications are river,mountain ,residential area, agriculture and forest. The operation of a one-to-many is explained in figure 2. Multiple loops of SVM operation are carried out for SVM operation. Here it can be seen that there are five categories and initially matching is carried out between category 1 and rest of the category (category 2, 3, 4 and 5 in our case).

The block diagram shows the classification of satellite images.

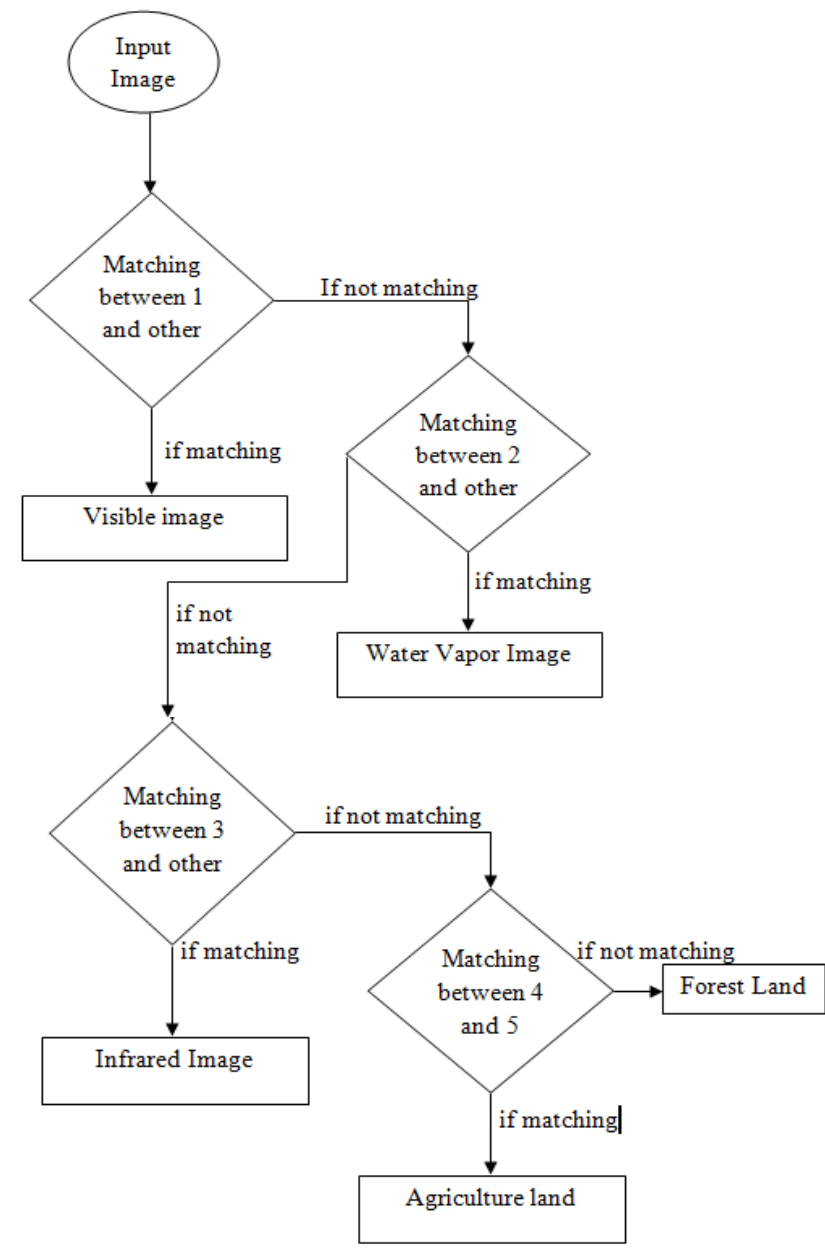

Figure 3. Block Diagram of SVM

Classification is carried out in two phases in SVM, namely testing phase and training phase. In the training phase the system is being taught using the features extracted and in testing phase, actual classification takes place. The feature extracted for the purpose is the average HSV value as the color feature of a region which is then converted to a semantic color name. In the testing phase, the input is classified by the trained SVM based on the features and the classifications are residential area, mountains, river, agricultural land and forest land.

\section{PROPOSED METHODOLOGY 4.1 Text Feature Extraction}

The purpose is to extract texture features from an image in which a RGB colored image is an input image and a multidimensional texture feature vector is an output image.

The following steps are followed:

Step1: Convert the RGB image into gray level.

Step2: Construct bank of 24 Gabor filters using the mother Gabor function with 4 scales and 6 orientations.

Step3: Apply Gabor filters on the gray level of the image by convolution.

Step4: Get the energy distribution of each of the 24 filters responses.

Step5: Compute the mean $\mu$ and the standard deviation Sigma of each energy distribution. 
Step6: Return the texture vector T consisting of 48 attributes calculated from step 5 .

\subsection{Color Feature Extraction}

In this system we used global color histograms in extracting the color features of images. The main issue regarding the use of color histograms for image retrieval involves the choice of color space, color space quantization into a number of color bins, where each bin represents a number of neighboring colors, and a similarity metric. HSV Color Space In the previous work, there is no optimum color space known for image retrieval, however certain color spaces such as HSV, Lab, and Luv have been found to be well suited for the content based query by color. We adopt to use the HSV (Hue, Saturation, and Value) color space for its simple transform from the RGB (Red, Green, Blue) color space, in which all the existing image formats are represented. The HSV color space is a popular choice for manipulating color, it is developed to provide an intuitive representation of color and to approximate the way in which humans perceive and manipulate color. RGB to HSV is a nonlinear, but reversible transformation. The hue $(\mathrm{H})$ represents the dominant spectral component (color in its pure form), as in red, blue, or yellow. Adding white to the pure color changes the color: the less white, the more saturated the color is. This corresponds to the saturation (S). The value (V) corresponds to the brightness of color. The hue (color) is invariant to the illumination and camera direction, and thus suitable for object recognition. Figure 8 shows the cylindrical representation of the HSV color space. The angle around the central vertical axis corresponds to "hue" denoted by the angle from 0 to 360 degrees, the distance from the axis corresponds to "saturation" denoted by the radius, and the distance along the axis corresponds to "lightness", "value" or "brightness" denoted by the height.

The HSV values of a pixel can be transformed from its RGB representation according to the following formulas:

$$
\begin{gathered}
\mathrm{H}=\operatorname{arctanSqrt}(3)(\mathrm{G}-\mathrm{B}) /(\mathrm{R}-\mathrm{G})+(\mathrm{R}-\mathrm{B}) \mathrm{S}=1-(\mathrm{Min} \\
\{\mathrm{R}, \mathrm{G}, \mathrm{B}\} / \mathrm{v})
\end{gathered}
$$

\section{IMPLEMENTATIONS AND RESULTS}

The proposed methodology consists of following three important phases.

First phase :-Features extraction.

Second phase:- Train database

Third phase :-Classification of input images.

All these phases are important to give the accurate results. In this proposed system, we start with the first phase called feature extraction. In this phase first the user selects the satellite image folder for the feature extraction and for feature extraction uses the Gabor filter properties of feature extraction and in which the features are extracted on the basis of texture and color.

The proposed technique is implemented in MATLAB on a system having 4 GB RAM and $2.6 \mathrm{GHz}$ Intel i-3 processor. The proposed system uses Gabor filter and support vector machine (SVM).

To implement the methodology the following steps are followed using a sample image.
Phase 1

In this system, first we input the satellite image.

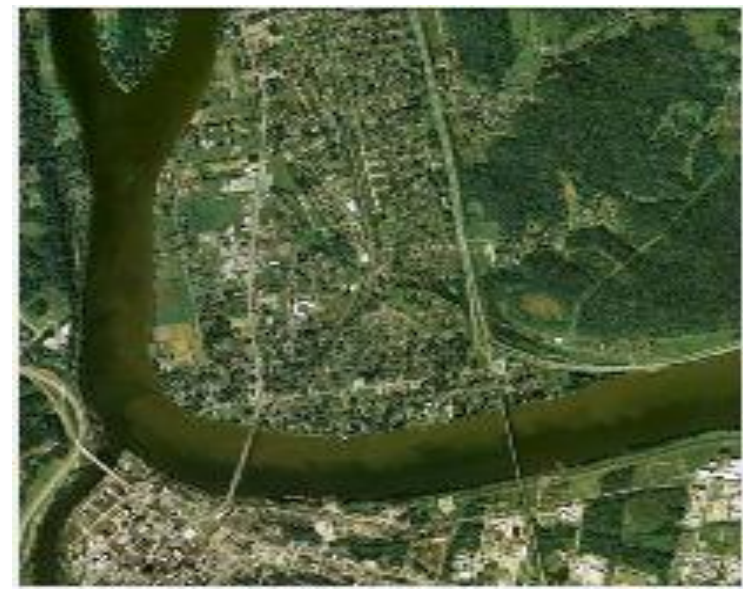

Figure 4: Satellite Image for processing

Then we find the grey scale of this input image figure 5 shows the grey scale image.

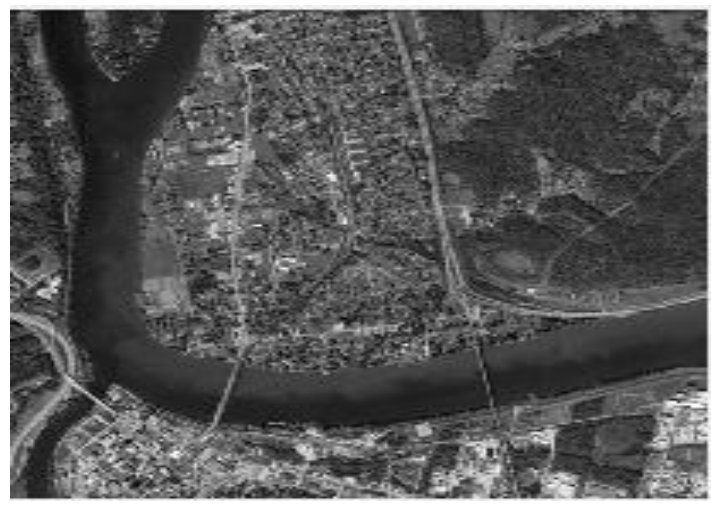

Figure 5: Grey scale of input image

Phase 2:- After this the image will convert from RGB to HSV for the feature extraction and feature selection. Figure 6 shows the converted RGB image to the HSV image.

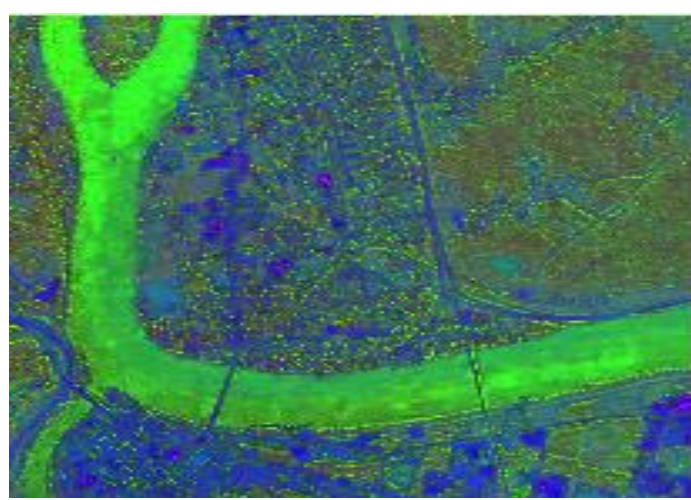

Figure 6: Convert RGB image to HSV

Phase 3:- Then the extracted features are filter with the Gabor filter and these features are trained with the help of support vector machine. First the magnitude of Gabor filter are find the real parts of Gabor filter are find by the system. 


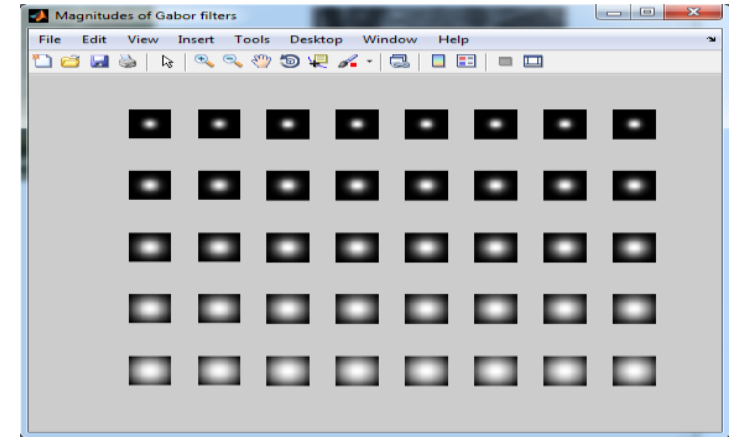

Figure 7: Magnitude of Gabor filter

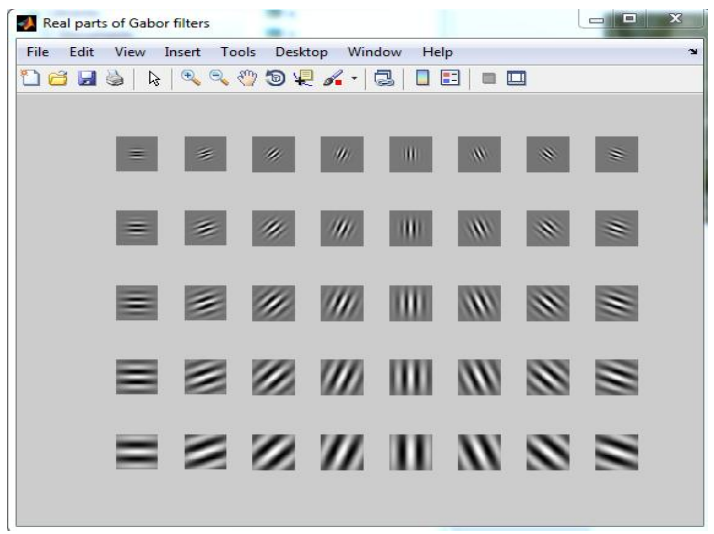

Figure 8: Real parts of Gabor Filter

The proposed technique is compared with DCT Gabor filter. Thus using the proposed technique the accuracy of the classification is better than DCT Gabor filter.

The figure 9 shows the accuracy results between DCT Gabor and proposed technique.

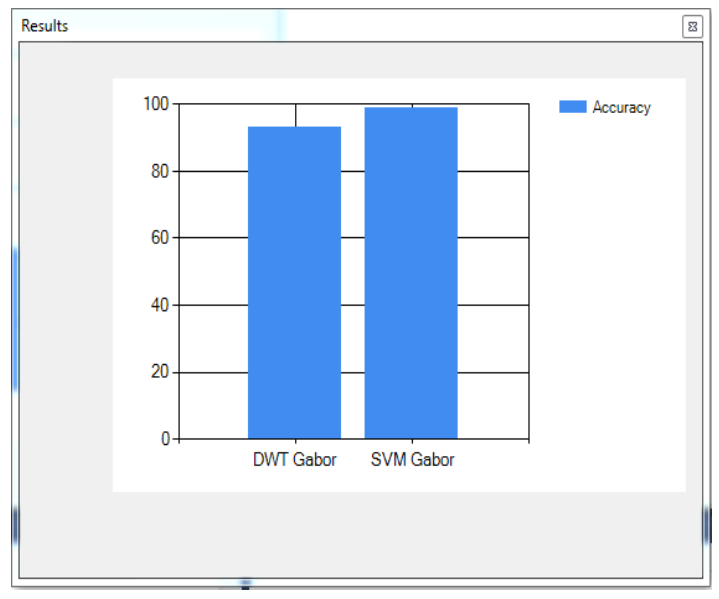

Figure 9: Result

\section{CONCLUSION}

The proposed satellite image classification to visible image, water vapor image, infrared image, agricultural and forest is carried out using Gabor filter with the feature extraction such as texture, color and shape and then train the database with the help of SVM training module and then classify the satellite image into three categories with the use of support vector machine (SVM) classifier. The accuracy of the proposed work is compared with the DCT Gabor filter. The result showed that the proposed technique has achieved better result reaching an accuracy of $98.5 \%$ for all satellite images.

\section{REFERENCES}

[1] J. Daugman, "High confidence visual recognition of persons by a test of statistical independence," IEEE Trans. on PAMI, vol. 25, no. 9, 1993.

[2] K. Messer and et al ., "Face authentication test on the BANCA database," in Int Conf on Pattern Recognition (ICPR), 2004.

[3] Jain, Y. Chen, and M. Demirkus, "Pores and ridges: Fingerprint matching using level 3 features," IEEE Trans. on PAMI, vol. 29, no. 1, 2007.

[4] M . Lades , J . C . Vorbr "uggen , J . Buhmann, J. Lange, C. von der Malsburg, R . P . W"urtz, and W. Konen, "Distortion invariant object recognition in the dynamic link architecture , " IEEE Trans. On Computers, vol. 42, pp. 300-311, 1993.

[5] Wiskott, J.-M. Fellous, N. Kr"uger, and C. von der Malsburg, "Face recognition by elastic bunch graph matching," IEEE Trans. on PAMI, vol. 19, 1997.

[6] J. G. Daugman, "Uncertainty relation for resolution in space, spatial frequency, and orientation optimized by two-dimensional visual cortical filters" Journal of the Optical Society of America A, vol. 2, no. 7, pp. 1160 1169, 1985.

[7] T. Serre, L. Wolf, S. Bileschi, M. Riesenhuber, and T. Poggio, "Object recognition with cortex-like mechanisms," IEEE Trans. on PAMI, vol. 29, no. 3, 2007.

[8] W. Niblack et al., "The QBIC Project," Proc. SPIE, vol. 1,908, pp. 173-181, Feb. 1993.

[9] J .G . Daugman, " Complete Discrete 2D Gabor Transforms by Neural Networks for Image Analysis and Compression," IEEE Trans.

[10] J. Ilonen, J.-K. Kamarainen, P. Paalanen, M. Hamouz, J. Kittler, and H. K"alvi"ainen, "Image feature localization by multiple hypothesis testing of Gabor features," IEEE Trans. on Image Processing, vol. 17, No. 3, pp. 311325,2008

[11] J. Ilonen, J.-K. Kamarainen, and H. K"alvi"ainen, "Fast extraction of multi-resolution gabor features," in 14th Int Conf on Image Analysis and Processing (ICIAP), 2007, pp. 481-486.

[12] E. Simoncelli, W. Freeman, E. Adelson, and D. Heeger "Shiftable Multiscale transforms," IEEE Transactions on Information Theory, vol. 38, no. 2, pp. 587-607, 1992.

[13] J . Sampo , J .-K . Kamarainen , M . Heilio , and H . Kalviainen "Measuring translation shiftability of frames ," Computers \& Mathematics with Applications, vol. 52, no. 6-7, pp. 1089-1098, 2006.

[14] M. Hamouz, J. Kittler, J.-K. Kamarainen, P. Paalanen, H. Kalviainen, and J. Matas, "Feature-based affineinvariant localization of faces," IEEE Trans. on PAMI vol. 27, no. 9, pp. 1490-1495, 2005.

[15] Cristianini , Nello and Shawe -Taylor , John, “ An Introduction to Support Vector Machines and other kernel based learning methods", Cambridge University Press, Cambridge, 2000. 